Article

\title{
Theoretical Investigation on Nearsightedness of Finite Model and Molecular Systems Based on Linear Response Function Analysis
}

\section{Yuki Mitsuta ${ }^{1}$, Shusuke Yamanaka ${ }^{1, *}$, Kizashi Yamaguchi ${ }^{2}$, Mitsutaka Okumura ${ }^{1}$ and Haruki Nakamura ${ }^{3}$}

1 Graduate School of Science, Osaka University, Machikaneyama 1-1, Toyoanaka, Osaka 560-0043, Japan; E-Mails: mitsutay13@chem.sci.osaka-u.ac.jp (Y.M.); ok@chem.sci.osaka-u.ac.jp (M.O.)

2 Institute for Nanoscience Design Center, Osaka University, Machikaneyama 1-3, Toyoanaka, Osaka 560-8531, Japan; E-Mail: yama@chem.sci.osaka-u.ac.jp

3 Institute for Protein Research, Osaka University, 3-2, Yamadaoka Suita, Osaka 565-0871, Japan; E-Mail: harukin@protein.osaka-u.ac.jp

* Author to whom correspondence should be addressed; E-Mail: syama@chem.sci.osaka-u.ac.jp; Tel.: +81-6-6850-5406; Fax: +81-6-6850-5550.

Received: 16 June 2014; in revised form: 31 July 2014 / Accepted: 14 August 2014 / Published: 29 August 2014

\begin{abstract}
We examined nearsightedness of electronic matter (NEM) of finite systems on the basis of linear response function (LRF). From the computational results of a square-well model system, the behavior of responses obviously depends on the number of electrons $(\mathrm{N})$ : as $\mathrm{N}$ increases, LRF, $\delta \rho(\mathrm{r}) / \delta \mathrm{v}\left(\mathrm{r}^{\prime}\right)$, decays rapidly for the distance, $\left|\mathrm{r}-\mathrm{r}^{\prime}\right|$. This exemplifies that the principle suggested by Kohn and Prodan holds even for finite systems: the cause of NEM is destructive interference among electron density amplitudes. In addition, we examined double-well model systems, which have low-lying degenerate levels. In this case, there are two types of LRF: the cases of the half-filled and of full-filled in low-lying degenerate levels. The response for the former is delocalized, while that of the later is localized. These behaviors of model systems are discussed in relation to the molecular systems' counterparts, $\mathrm{H}_{2}, \mathrm{He}_{2}{ }^{2+}$, and $\mathrm{He}_{2}$ systems. We also see that NEM holds for the dissociated limit of $\mathrm{H}_{2}$, of which the mechanism is similar to that of the insulating state of solids as suggested by Kohn. We also examined LRF of alanine tripeptide system as well as butane and butadiene molecules, showing that NEM of the polypeptide system is caused by $\mathrm{sp}_{3}$ junctions at $\mathrm{C} \alpha$ atoms that prevent propagation of amplitudes of LRF, which is critically different from that of NEM for finite and infinite homogeneous systems.
\end{abstract}


Keywords: nearsightedness of electronic matter; linear response function; finite systems

\section{Introduction}

Karplus, Warshel and Levitt won the 2013 Novel Prize in Chemistry for developing the multiscale models, i.e., quantum mechanics/molecular mechanics (QM/MM) models, for complex chemical systems $[1,2]$. In the QM/MM models, chemically active sites are described with QM methods while peripheral regions such as protein environments in enzymes and solvents in liquids with MM methods where the electronic structures of them are replaced by classical point charges. In such approximations, we assume that the artificial Coulomb potentials due to the point charges do not deteriorate description of electronic structures of the QM sites. In other words, this type of approaches relies on nearsightedness of electronic matter (NEM) as proposed by Kohn and Prodan [3,4], the fact that the changes of electric potentials at any points that are far enough from a specific point do not affect significantly electronic properties at the point. They proved that NEM holds for some infinite ordered and nonordered systems. Kohn has also analyzed the Mott insulating states to show that the low-lying states can be described with a sum of "localized wavefunctions", which is another type of NEM [5]. A noteworthy point is that they deliberately excluded small systems of a few electrons from a list of substances for which NEM holds. This implies that the simplest molecule system, hydrogen molecule, is not included the list. The problem is whether NEM holds for molecular systems: as described above, quantum chemists using the QM/MM models also assume that NEM holds for large molecular systems. If so, as the number of electrons in molecules increases from two of hydrogen molecule, which size does NEM start to hold for? What is the difference between hydrogen molecule and a large molecule for which NEM holds? To begin with, what is the reason that NEM holds for the large molecular systems? Is it the same reason that was presented by Prodan and Kohn for infinite systems [3-5]?

In order to provide answers to these questions, we examined two types of systems: the first one is simple model systems such as electrons in square-well potential and electrons in harmonic oscillator potential. These systems are simple models for molecular systems, but without the intrinsic structure due to the existence of atoms such as atomic orbitals and chemical bonds. This simplicity of finite model systems is similar to that of the infinite systems that were presented by Prodan and Kohn [4].

The second one is simple molecular systems with using ab initio density functional computational results, which are directly related to the QM/MM applications. The comparison among LRFs $\left(\delta \rho(\mathbf{r}) / \delta v\left(\mathbf{r}^{\prime}\right)\right)$ for these two types of systems reveals that the propagations of density deviations $(\delta \rho(\mathbf{r}))$ due to virtual perturbations $\left(\delta \mathrm{v}\left(\mathbf{r}^{\prime}\right)\right)$ are the results of different type of effects.

\section{Theoretical Background}

In the original theory of NEM, Prodan and Kohn state that NEM is not an aspect of linear or nonlinear response to external perturbations. At the same time, they also state that NEM does not exclude them. In fact, they also analyzed density changes due to perturbations, which is similar to linear response function, $\delta \rho(\mathbf{r}) / \delta \mathrm{v}\left(\mathbf{r}^{\prime}\right)$. Density is the most important quantity in electronic structure theory: in density functional theory, it determines all of other electronic properties uniquely [6,7]. 
Also, two leading terms in electronic energies, nuclear-attraction and classical Coulomb repulsion terms explicitly depend on density. Thus, the change of density directly leads to the change of energy of the system. In addition, according to the Feynman's electrostatic theorem [8], forces acting on nuclei depend on interactions among nuclei and electron density of the system. Because the movement of nuclei in either molecular dynamics or geometry optimizations is determined by the forces, density also determines dynamical behaviors of the system. Therefore, the density change results in the change of both static and dynamic behavior of the system. This is the reason why the density is important in various fields from fundamental chemistry to biological and material sciences (for a comprehensive review, see reference [9]). For the perturbation, $\delta \mathrm{v}\left(\mathbf{r}^{\prime}\right)$, the change of density is given by:

$$
\delta \rho(\mathbf{r})=\int \mathrm{d} \mathbf{r}^{\prime} \frac{\delta \rho(\mathbf{r})}{\delta \mathrm{v}\left(\mathbf{r}^{\prime}\right)} \delta \mathrm{v}\left(\mathbf{r}^{\prime}\right)
$$

A well-known example is a phenomenon that is called "Friedel oscillation" of an impurity problem for metals [10-13]. In this case, $\delta v\left(\mathbf{r}^{\prime}\right)$ is the perturbation due to the magnetic impurities that causes density polarizations in metals around the impurities. This phenomenon is well described with the linear response theory under the random phase approximation for a homogenous electron gas (HEG) model [14]: The analytical form of density polarization is given by:

$$
\delta \rho(r)=-\frac{\mathrm{q}_{\mathrm{TF}}^{2}}{4 \pi \mathrm{r}} \mathrm{e}^{-\mathrm{q}_{\mathrm{TF}} \mathrm{r}}+\frac{1}{\pi} \frac{\mathrm{q}_{\mathrm{TF}}^{2} / \mathrm{k}_{\mathrm{TF}}^{2}}{\left(4+\mathrm{q}_{\mathrm{TF}}^{2} /\left(2 \mathrm{k}_{\mathrm{TF}}^{2}\right)\right)^{2}} \frac{\cos \left[2 \mathrm{k}_{\mathrm{F}} \mathrm{r}\right]}{\mathrm{r}^{3}}
$$

here, $\mathrm{q}_{\mathrm{TF}}$ is the inverse of the Thomas-Fermi screening length, $\mathrm{k}_{\mathrm{F}}$ is the Fermi wavenumber, and $\mathrm{r}$ is the distance from the point charge that mimics the impurity. The first term is the monotonically exponential decay term, and the second term causes the decaying "oscillation" term around the impurity, which was observed as NMR signals $[11,12]$ and directly as scanning probe images about a decade ago [13]. A noteworthy point is that the change of density is a function only of the distance from the point charge because the HEG system is homogeneous and isotropic. If the system is well approximated as a HEG system, the above Equation (2) is effective for describing the response to virtual perturbations. However, because the molecular systems are neither homogeneous nor isotropic, responses to perturbations must strongly depend on both the response point and the point to which the perturbation is applied. In that case, it is essential to consider the linear response function (LRF) in the matrix form that is given by:

$$
\frac{\delta \rho(\mathbf{r})}{\delta \mathrm{v}\left(\mathbf{r}^{\prime}\right)}=2 \sum_{\mathrm{i}}^{\mathrm{Nocc}} \sum_{\mathrm{j}}^{\mathrm{unocc}} \frac{\psi_{\mathrm{i}}(\mathbf{r}) \psi_{\mathrm{i}}\left(\mathbf{r}^{\prime}\right) \psi_{\mathrm{j}}(\mathbf{r}) \psi_{\mathrm{j}}\left(\mathbf{r}^{\prime}\right)}{\varepsilon_{\mathrm{i}}-\varepsilon_{\mathrm{j}}}
$$

using the straightforward perturbation treatment. Here $\psi_{\mathrm{i}}(\mathbf{r})$ and $\psi_{\mathrm{j}}(\mathbf{r})$ are occupied and unoccupied orbitals respectively, and $\varepsilon_{\mathrm{i}}$ and $\varepsilon_{\mathrm{j}}$ are the corresponding orbital energies. In addition, double summations in the right side of Equation (3) run over occupied orbitals (Nocc) (i) and unoccupied orbitals (j). Because we here assume that the closed-shell many-electrons systems, the factor, 2, appears in the right side. We would like to emphasize here that LRF is a function of both the response point (r) and the perturbed point $\left(\mathbf{r}^{\prime}\right)$, which is critically different from the case of the HEG system. It is also noteworthy that the integral of LRF over the space is zero, i.e., $\int \delta \rho(\mathbf{r}) / \delta \mathrm{v}\left(\mathbf{r}^{\prime}\right) \mathrm{d} \mathbf{r}=0$, because the number of electrons is conserved. 
During the past decades, LRFs of molecules has been computed in relation to the chemical reactivities [15-22]. In particular, an important relation is the Berkowitz-Parr relation [17] as given by:

$$
\frac{\delta \rho(\mathbf{r})}{\delta v\left(\mathbf{r}^{\prime}\right)}=-\mathrm{s}\left(\mathbf{r}, \mathbf{r}^{\prime}\right)+\frac{\mathrm{s}(\mathbf{r}) \mathrm{s}\left(\mathbf{r}^{\prime}\right)}{\mathrm{S}}
$$

here $\mathrm{S}, s(\mathrm{r})$, and $s\left(\mathrm{r}, \mathrm{r}^{\prime}\right)$ are softness, local softness, and the kernel of the local softness, respectively, all of which are essential properties in important applications of conceptual DFT to the hard and soft acid and bases (HSAB) principle. This type of theories provides new viewpoints and fruitful discussions that link between DFT and chemistry (for instance, see a comprehensive review, reference [16]), but we will not step into this fruitful field of softness and their related properties any further. Instead, we focus our attention on whether and how the nearsightedness holds for the finite model systems as well as molecular systems. Of course, the reactivities of molecules are closely related to the issue of nearsightedness of molecules. In particular, the substituent effects such as inductive and resonance effects are not localized on the few sites, for which NEM does not holds [19]. Our study is to examine NEM of finite systems on the basis of LRF analyses of both model systems and molecular systems. In the following, we start from more simple examples using model systems that are well-known in the primer of the Quantum Mechanics [23]. Then we proceed to the molecular systems in order to make a comparison between model systems and molecular systems on the origins of nearsightedness.

\section{Results and Discussion}

\subsection{Model Systems}

\subsubsection{An Infinite Square Well Potential System}

First, we consider many electrons systems in one-dimensional (1-D) square well potentials. In this case, the Schrödinger Equation is given by:

$$
-\frac{\hbar^{2}}{2 \mathrm{M}_{\mathrm{e}}} \nabla^{2} \psi_{\mathrm{n}}(\mathrm{r})+\mathrm{U}(\mathrm{r}) \psi_{\mathrm{n}}(\mathrm{r})=\mathrm{E}_{\mathrm{n}} \psi_{\mathrm{n}}(\mathrm{r})
$$

where $M_{e}$ is mass of electron and $U(r)$ is the square well potential given by the following:

$$
U(r)=\left\{\begin{array}{cc}
0 & 0 \leq r \leq 1 \\
\infty & r<0 \text { or } 1<r
\end{array}\right.
$$

The electrons are confined in this finite 1-D space. The eigenfunctions and those eigenvalues are respectively:

$$
\psi_{\mathrm{n}}(\mathrm{r})=\sqrt{2} \sin (\mathrm{n} \pi \mathrm{x})
$$

and:

$$
\mathrm{E}_{\mathrm{n}}=\frac{\mathrm{n}^{2} \hbar^{2} \pi^{2}}{2 \mathrm{M}_{\mathrm{e}}}
$$

We ignore electron interactions. Thus, the linear response function is given by: 


$$
\frac{\delta \rho(r)}{\delta v\left(r^{\prime}\right)}=\frac{16 M_{e}}{\hbar^{2} \pi^{2}} \sum_{n}^{N o c c} \sum_{m}^{u n o c c} \frac{\sin (n \pi r) \sin \left(n \pi r^{\prime}\right) \sin (m \pi r) \sin \left(m \pi r^{\prime}\right)}{n^{2}-m^{2}}
$$

Our focus is on the dependency of $\delta \rho(r) / \delta v\left(r^{\prime}\right)$ on $\left|r-r^{\prime}\right|$. Since the factor is essentially irrelevant to the dependency, we analyzed:

$$
\frac{\delta \rho(r)}{\delta \mathrm{v}\left(\mathrm{r}^{\prime}\right)} \sim \sum_{\mathrm{n}}^{\mathrm{Nocc}} \sum_{\mathrm{m}}^{\mathrm{unocc}} \frac{\sin (\mathrm{n} \pi \mathrm{r}) \sin \left(\mathrm{n} \pi \mathrm{r}^{\prime}\right) \sin (\mathrm{m} \pi \mathrm{r}) \sin \left(\mathrm{m} \pi \mathrm{r}^{\prime}\right)}{\mathrm{n}^{2}-\mathrm{m}^{2}}
$$

The calculations of the LRF given by Equation (10) were done with using Mathematica Ver. 9 [24]. We determine the upper limit of the summation over the unoccupied orbitals in Equation (10) in the following manner: We calculate the integral of the square of each term; and if that value is lower than $10^{-8}$; i.e.,:

$$
\int d r \int d r^{\prime}\left(\frac{\sin (n \pi r) \sin \left(n \pi r^{\prime}\right) \sin (m \pi r) \sin \left(m \pi r^{\prime}\right)}{n^{2}-m^{2}}\right)^{2} \leq 10^{-8}
$$

then the summation is truncated at the value $\mathrm{m} \equiv \mathrm{M}_{\text {truncated }}$ :

$$
\frac{\delta \rho(\mathrm{r})}{\delta \mathrm{v}\left(\mathrm{r}^{\prime}\right)} \sim \sum_{\mathrm{n}}^{\mathrm{Nocc}} \sum_{\mathrm{m}}^{\mathrm{M}_{\text {truncated }}} \frac{\sin (\mathrm{n} \pi \mathrm{r}) \sin \left(\mathrm{n} \pi \mathrm{r}^{\prime}\right) \sin (\mathrm{m} \pi \mathrm{r}) \sin \left(\mathrm{m} \pi \mathrm{r}^{\prime}\right)}{\mathrm{n}^{2}-\mathrm{m}^{2}}
$$

In the following, we employed this Equation to estimate LRF. Figure 1 shows plots of the calculated two-dimensional LRFs for various numbers of occupied orbitals (Nocc). LRF is symmetric, i.e., $\delta \rho(\mathrm{r}) / \delta \mathrm{v}\left(\mathrm{r}^{\prime}\right)=\delta \rho\left(\mathrm{r}^{\prime}\right) / \delta \mathrm{v}(\mathrm{r})$, as is obvious from Equation (10).

Figure 1. Plots of linear response functions of the infinite square-well potential system for various numbers of occupied orbitals (Nocc). (a) Nocc $=1$; (b) Nocc $=2$; (c) Nocc = 3; (d) Nocc = 4; (e) Nocc = 5; (f) Nocc = 10; (g) Nocc = 20; (h) Nocc = 35; (i) Nocc = 50.

(a)

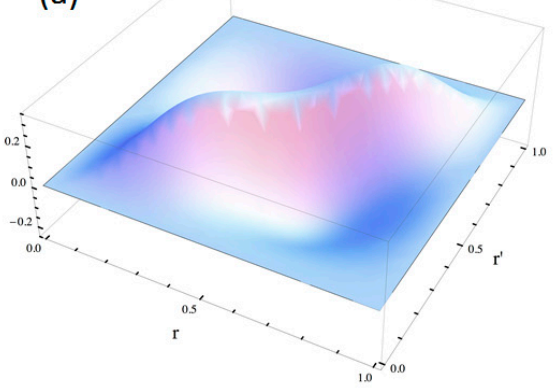

(d)

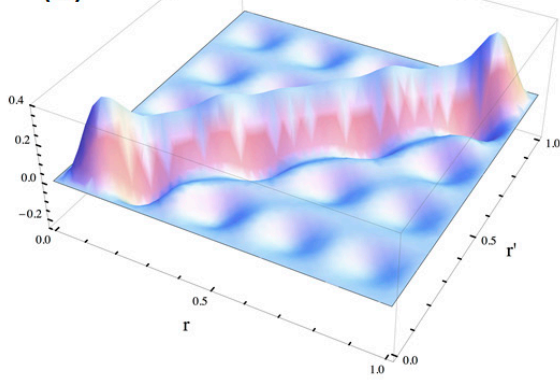

(b)

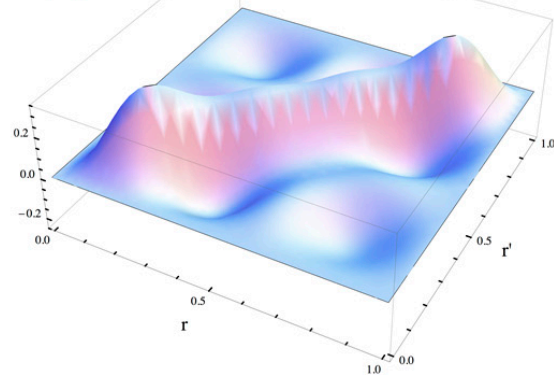

(e)

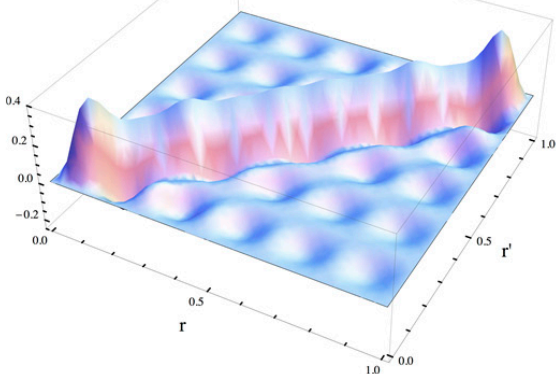

(c)

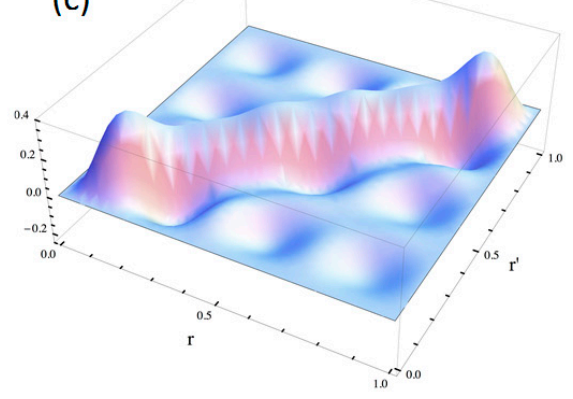

(f)

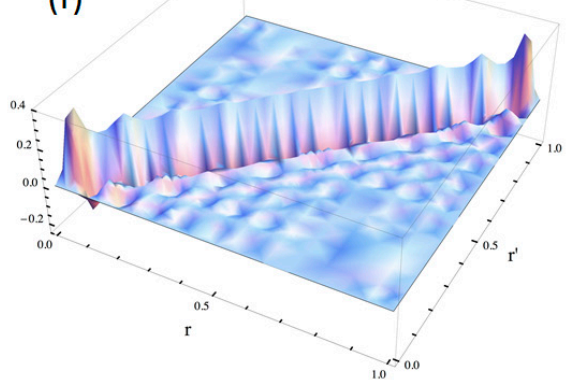


Figure 1. Cont.
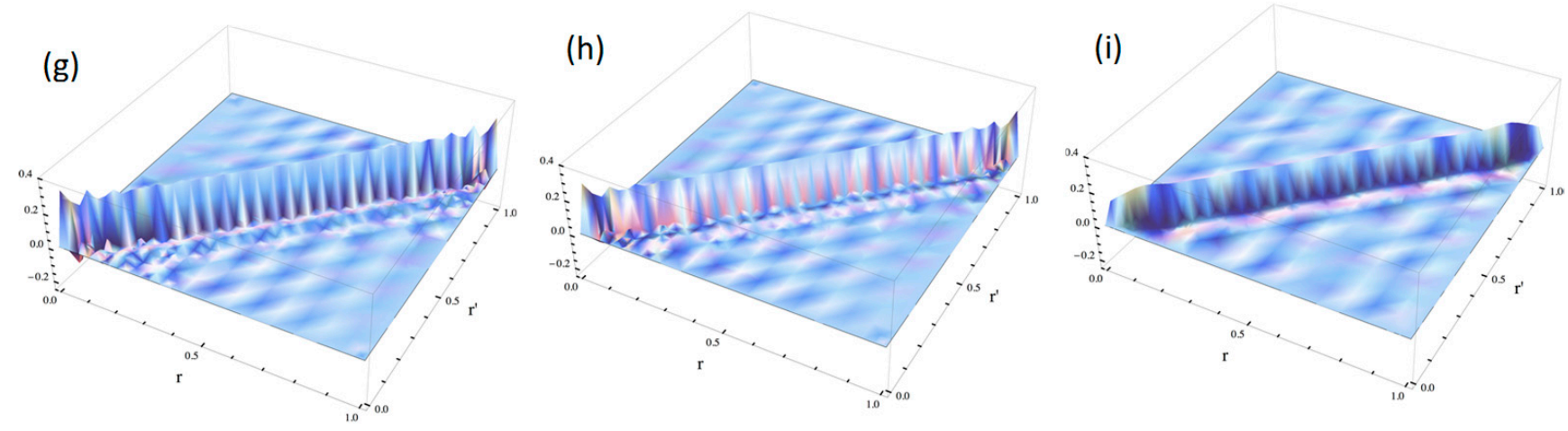

In order to make the meanings of the plots clear, we also plotted $\delta \rho(r) / \delta v\left(r^{\prime}\right)$ for several specific points, $\mathrm{r}^{\prime}$, to which the virtual perturbations $\delta \mathrm{v}\left(\mathrm{r}^{\prime}\right)$ are applied, in Figure $2($ Nocc $=1)$ and Figure 3 $($ Nocc $=50)$. At the site around the point where the perturbation is applied, the density decreases, while it increases at other regions to conserve the number of electrons.

Figure 2. Plots of $\operatorname{dr}(\mathrm{r}) / \mathrm{dv}\left(\mathrm{r}^{\prime}\right)$ where virtual perturbations are applied to specific points $\left(\mathrm{r}^{\prime}\right)$ indicated by arrows for the Nocc $=1$ case. (a) $r^{\prime}=0.5 ;(\mathbf{b}) r^{\prime}=0.6 ;$ (c) $r^{\prime}=0.8$.

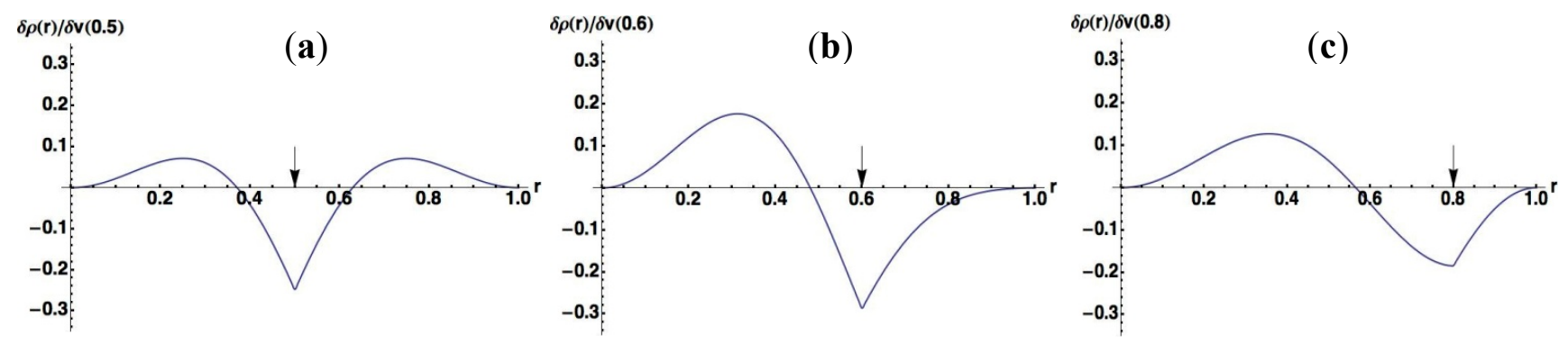

Figure 3. Plots of $\operatorname{dr}(\mathrm{r}) / \mathrm{dv}\left(\mathrm{r}^{\prime}\right)$ where virtual perturbations are applied to specific points $\left(\mathrm{r}^{\prime}\right)$ indicated by arrows for the Nocc $=50$ case. (a) $r^{\prime}=0.5 ;(\mathbf{b}) r^{\prime}=0.6 ;(\mathbf{c}) r^{\prime}=0.8$.

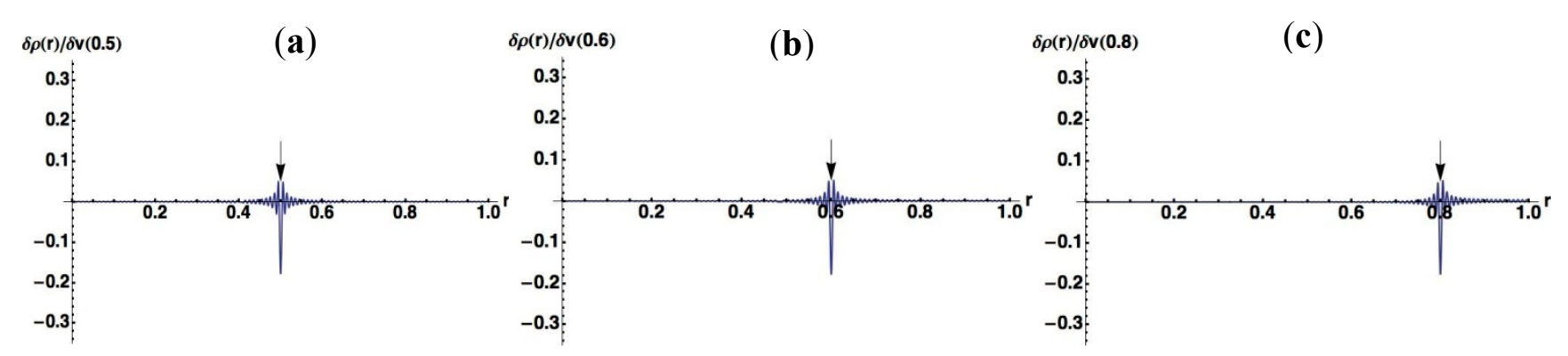

A noteworthy point for Figure 2 is that the change of density due to the perturbation spreads over whole the region in $1 \mathrm{D}$ space for Nocc $=1$. On the other hand, we can see from Figure 3 that the effects due to perturbations are localized for Nocc $=50$. Figures 2 and 3 present remarkable contrast between Nocc $=1$ and Nocc $=50$ : we can state that NEM holds for Nocc $=50$ case, but not for Nocc $=1$ case. We can see from the plots of Figure $1 \mathrm{a}-\mathrm{i}$ that, as Nocc increases, $\delta \rho(r) / \delta v\left(r^{\prime}\right)$ decays rapidly for the distance, $\left|r-r^{\prime}\right|$. This is consistent with the fact that NEM is a result of destructive interference among density amplitudes suggested by Prodan and Kohn [4]. We also performed a similar analysis 
for harmonic oscillator systems, and presented the results in the supporting Section S1: the conclusion is similar to that from results of this square well potential.

\subsubsection{Double-well Potential Systems}

Next, we consider many electrons systems in 1-D double-well potentials which is given by:

$$
\mathrm{U}(\mathrm{r})=\left\{\begin{array}{cc}
0 & -2 \leq \mathrm{r} \leq-1 \text { or } 1 \leq \mathrm{r} \leq 2 \\
\mathrm{~V}_{0} & -1 \leq \mathrm{r} \leq 1 \\
\infty & \mathrm{r}<-2 \text { or } 2<\mathrm{r}
\end{array}\right.
$$

Note that there is a barrier in the center of the system. In this case, there are two types of eigenfunctions: symmetric and antisymmetric functions. Here, we examined two values, 25 and 100 for $\mathrm{V}_{0}$, in order to confirm the effects of the height of the barrier. The solutions are described in Section S2 in the Supplementary Materials. A noteworthy point is that, in contrast to the single-well case examined in Section 3.1.1, there are pairs of symmetric and antisymmetric states as shown in the schematic illustrations of Figure 4a,b. Eigenfunctions of each pair are quasi-degenerate under $\mathrm{E}_{\mathrm{k}_{\mathrm{n}}^{ \pm}}<\mathrm{V}_{0}$, while the quasi-degeneracy is lifted for $\mathrm{E}_{\mathrm{k}_{\mathrm{n}}^{ \pm}}>\mathrm{V}_{0}$ :

Figure 4. Energy level of double-well potential systems. (a) $\mathrm{V}_{0}=25$. (b) $\mathrm{V}_{0}=100$.

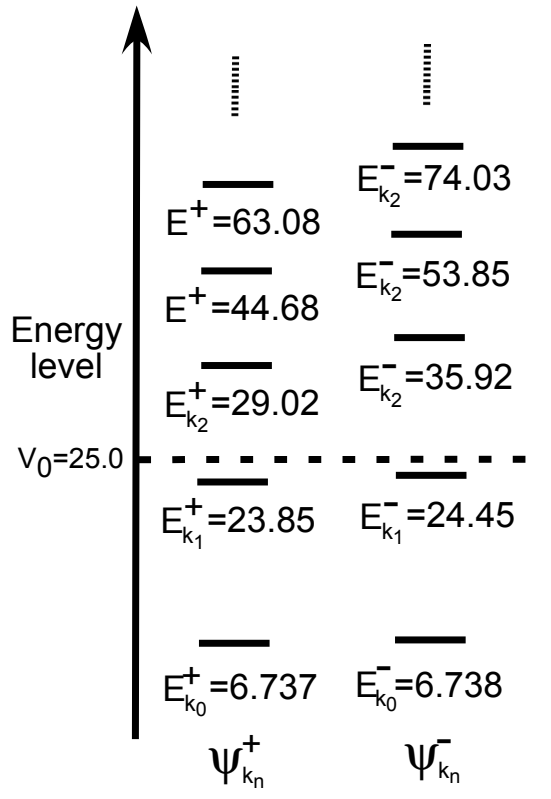

(a)

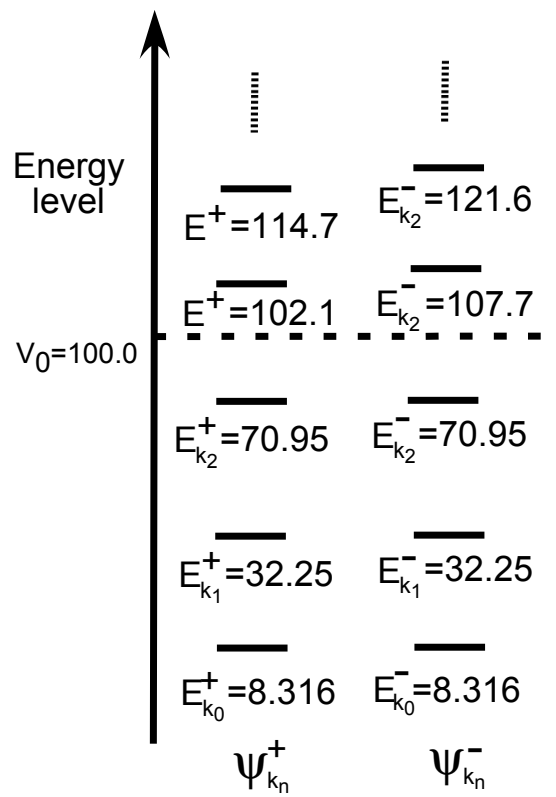

(b)

The numbers of pairs with $\mathrm{E}_{\mathrm{k}_{\mathrm{n}}^{ \pm}}<\mathrm{V}_{0}$ are two and three for $\mathrm{V}_{0}=25$ and $\mathrm{V}_{0}=100$, respectively, as shown in Figure 4a,b. The exact eigenvalues are listed in the Section S2 in the Supplementary Materials. Now we performed the LRF analysis in a manner similar to that described in Section 3.1.1. We plotted LRFs for various numbers of Nocc for $\mathrm{V}_{0}=25$ in Figure 5 and $\mathrm{V}_{0}=100$ in Figure 6. For the plane of each plot, lines indicate the borders that divide the 1D space into three regions, $-2 \leq \mathrm{r}\left(\mathrm{r}^{\prime}\right) \leq-1,-1 \leq \mathrm{r}\left(\mathrm{r}^{\prime}\right) \leq 1$, and $1 \leq \mathrm{r}\left(\mathrm{r}^{\prime}\right) \leq 2$. For $\mathrm{V}_{0}=25$, there are four orbitals that have lower energies than the height of the barrier, $\mathrm{V}_{0}$. We can see from Figure $5 \mathrm{a}, \mathrm{b}$ that there are significant nonlocal responses for Nocc $=1$ and Nocc $=3$ (Figure 5a,c), while there is no nonlocal responses for 
Nocc $=2$ and Nocc $=4$ (Figure 5b,d). For Nocc $=5$ shown in Figure 5e, an amplitude appears in the central region, $-1 \leq \mathrm{r} \leq 1$, that corresponds to the barrier region, because the electrons having energies higher than $\mathrm{V}_{0}=25$ exist. An interesting point is that the form of the amplitude within the central region, $-1 \leq \mathrm{r} \leq 1$, in Figure 5e is similar to that in Figure 1a. This nonlocality of the central region reduces as Nocc increases as shown in Figure $5 \mathrm{f}-\mathrm{i}$. The LRF for Nocc $=50$ shown in Figure $5 \mathrm{i}$ is similar to that of the single-well potential shown in Figure 1i, implying that NEM is a result of destructive interference among density amplitudes also in this case. A noteworthy point is that NEM seems to hold also for small numbers of electrons as shown in Figure 5b,d, which must be caused by a different mechanism. Similar results are observed in Figure 6. In particular, "NEM of small numbers of electrons" is also observed in Figure $6 \mathrm{~b}-\mathrm{f}$, because there are three pairs of quasi-degenerate levels as shown in Figure $4 b$.

Figure 5. Plots of linear response functions of the double-well potential system $\left(\mathrm{V}_{0}=25\right)$ for various numbers of occupied orbitals (Nocc). (a) Nocc $=1 ;(\mathbf{b})$ Nocc $=2 ;$ (c) Nocc $=3$; (d) $\operatorname{Nocc}=4 ;($ e) Nocc = 5; (f) Nocc = 10; (g) Nocc = 20; (h) Nocc = 35; (i) Nocc = 50.
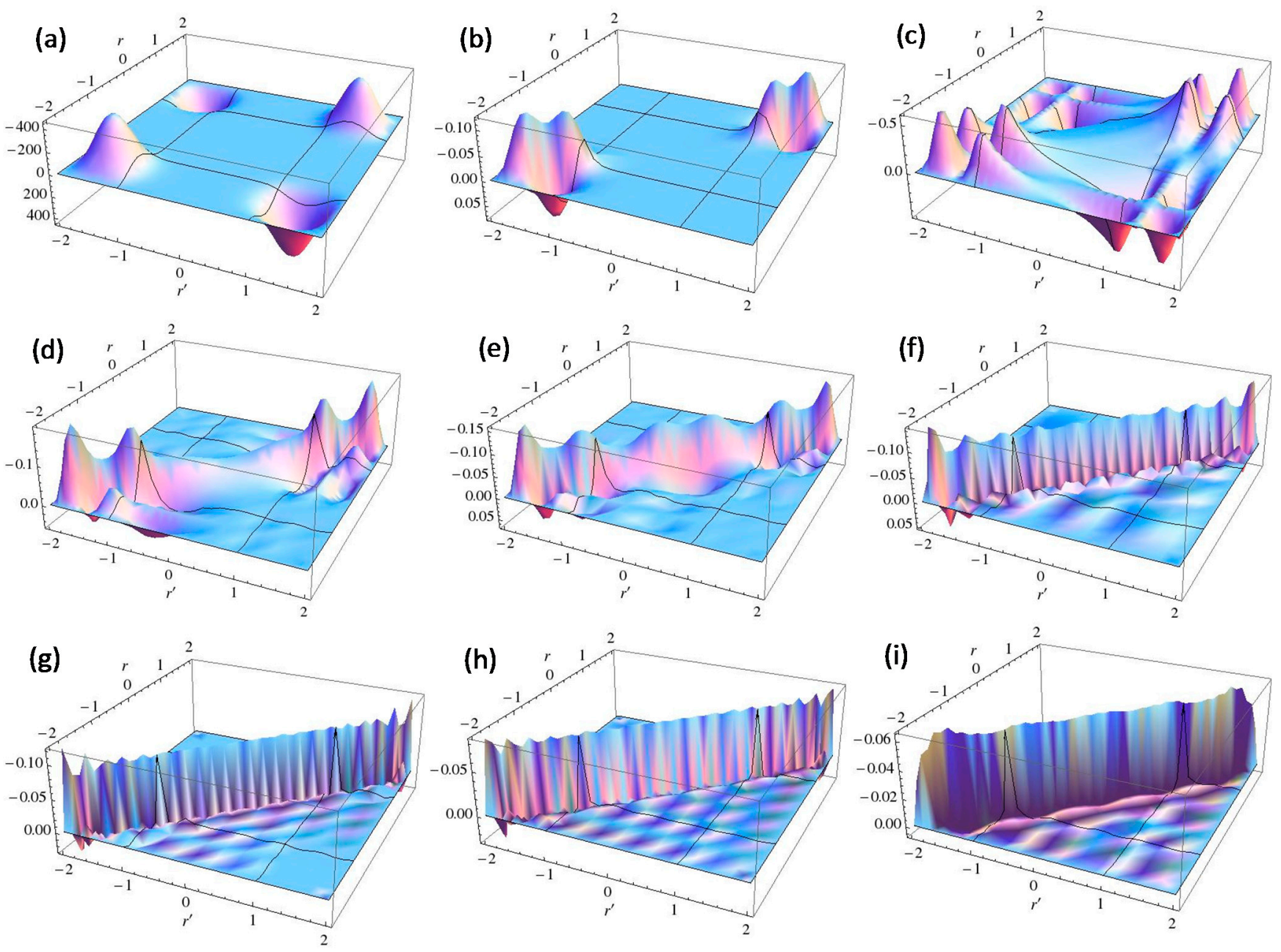

Judging from these results, we could conjecture that, if there are fully occupied orbitals that are degenerate each other, NEM holds even for a few electrons systems. We will confirm that this conjecture holds also for molecular systems in the next section. 
Figure 6. Plots of linear response functions of the double-well potential system $\left(\mathrm{V}_{0}=100\right)$ for various numbers of occupied orbitals (Nocc). (a) Nocc $=1 ;(\mathbf{b})$ Nocc $=2 ;$ (c) Nocc $=3$; (d) $\operatorname{Nocc}=4 ;($ e) $\operatorname{Nocc}=5 ;($ f) Nocc $=6 ;($ g) Nocc $=7 ;($ h $)$ Nocc $=25 ;$ (i) $\operatorname{Nocc}=50$.
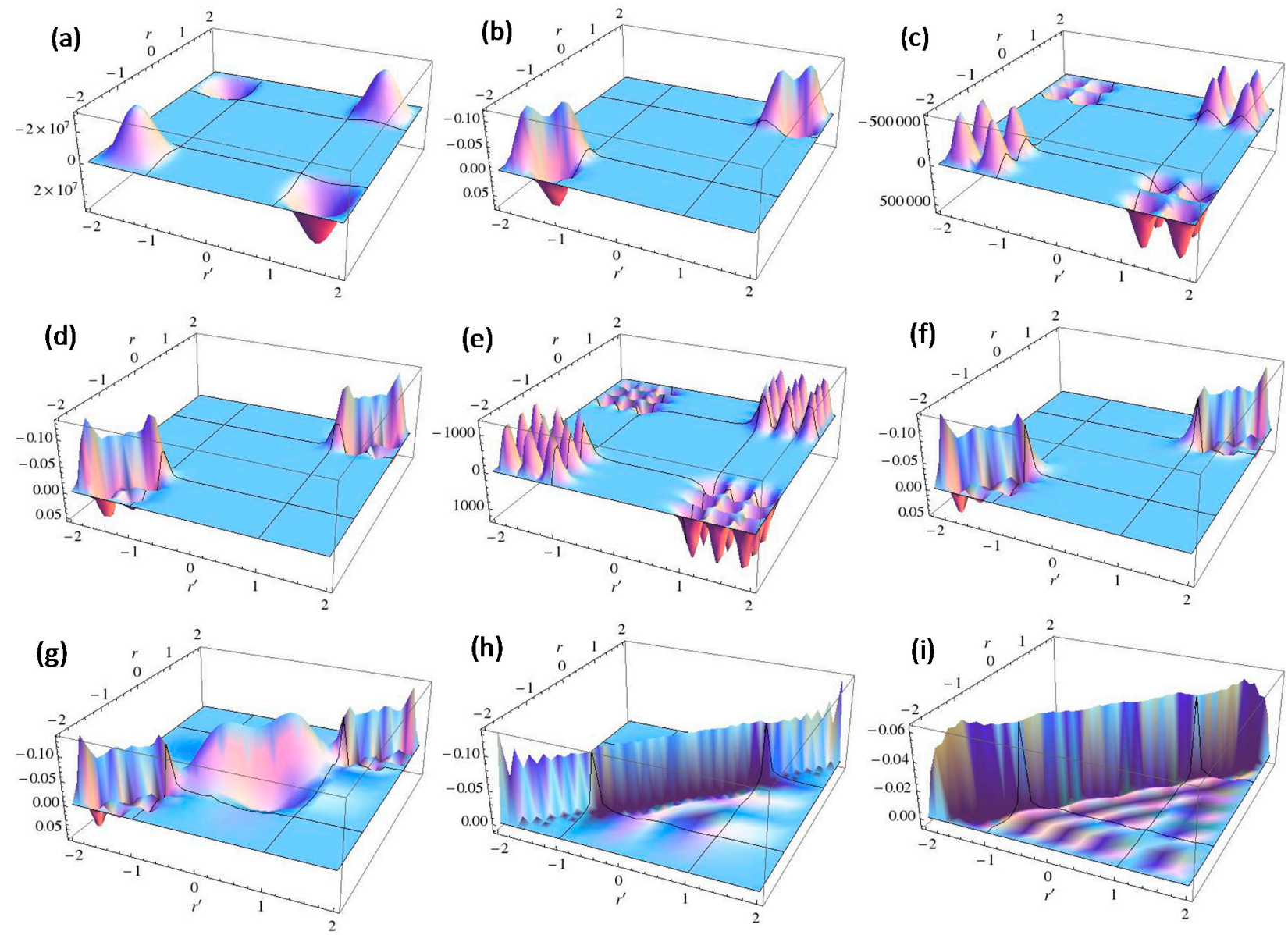

\subsection{Molecular Systems}

\subsubsection{Molecular Systems' Counterparts for the Model Systems}

We would like to proceed to molecular systems. First we would like to examine molecular system's counterparts for the model systems described above. For this purpose, we computed LRF of molecular systems in terms of linear combination of atomic orbitals (LCAO) with using ab initio Kohn-Sham density functional solutions $[25,26]$ :

$$
\frac{\delta \rho(\mathbf{r})}{\delta \mathrm{v}(\mathrm{X})}=2 \sum_{\mathrm{i}}^{\mathrm{occ}} \sum_{\mathrm{j}}^{\mathrm{unocc}} \sum_{\mu}^{\mu \in \mathrm{X}} \int \mathrm{d} \mathbf{r}^{\prime} \frac{\psi_{\mathrm{i}}(\mathbf{r}) \psi_{\mathrm{i}}\left(\mathbf{r}^{\prime}\right) \psi_{\mathrm{j}}(\mathbf{r}) \chi_{\mu}\left(\mathbf{r}^{\prime}\right)}{\varepsilon_{\mathrm{i}}-\varepsilon_{\mathrm{j}}} \mathrm{C}_{\mathrm{j} \mu}
$$

here $\chi_{\mu}$ is a $\mu$-th atomic orbital (AO), and $\mathrm{C}_{\mathrm{j} \mu}$ is a molecular coefficient of $\mathrm{j}$-th $\mathrm{MO}$ and $\mu$-th $\mathrm{AO}$. We performed the following calculations of LRF for molecular systems with using an extended version of GAMESS [27], in which our module to compute LRF is implemented. The details are described in references $[25,26]$. In the following, B3LYP and 6-31G** are used for exchange-correlation functional and basis set respectively [28].

A first example is hydrogen molecule with fixing the interatomic distance $(\mathrm{R})$ at the equilibrium value $(0.72 \AA)$. Figure $7 \mathrm{a}$ shows the equivalue surface of the LRF, $\delta \rho(\mathbf{r}) / \delta \mathrm{v}(\mathrm{X})$. Here $\delta \mathrm{v}(\mathrm{X})$ is a virtual 
repulsive perturbation applied to the right-atom. The blue surface indicates $\delta \rho(\mathbf{r}) / \delta \mathrm{v}(\mathrm{X})=-0.01$ and the red surface $\delta \rho(\mathbf{r}) / \delta \mathrm{v}(\mathrm{X})=+0.01$. The antisymmetric feature of LRF of $\mathrm{H}_{2}$ can be explained as follows: first, we could approximate the LRF of $\mathrm{H}_{2}$ as:

$$
\frac{\delta \rho(\mathbf{r})}{\delta \mathrm{v}\left(\mathbf{r}^{\prime}\right)} \cong 2 \frac{\psi_{\mathrm{HOMO}}(\mathbf{r}) \psi_{\mathrm{HOMO}}\left(\mathbf{r}^{\prime}\right) \psi_{\mathrm{LUMO}}(\mathbf{r}) \psi_{\mathrm{LUMO}}\left(\mathbf{r}^{\prime}\right)}{\varepsilon_{\mathrm{HOMO}}-\varepsilon_{\mathrm{LUMO}}}
$$

because the orbital energy of the next LUMO is 0.569 Hartree, which is higher than that of LUMO $(0.107$ Hartree) and that of HOMO $(-0.436$ Hartree). Thus, the spatial distribution of $\delta \rho(\mathbf{r}) / \delta \mathrm{v}(\mathrm{X})$ is determined with $\psi_{\text {номо }}(\mathbf{r}) \psi_{\text {LUмо }}(\mathbf{r})$, which is obviously an antisymmetric function, resulting in the antisymmetric LRF shown in Figure 7a. This LRF is obviously parallel with that of two electrons in the square well potential shown in Figure 2b,c: the density at the left side increases and that at the right side decreases. Obviously, NEM does not hold for this case as Prodan and Kohn excluded few-electron systems from the target of NEM [4].

A second example is $\mathrm{He}_{2}{ }^{2+}$ with the interatomic distance, $\mathrm{R}=3.0 \AA$. Because this interatomic distance is too far to form the covalent-bonding between He atoms, we can consider that the vacant space between atoms is the energy barrier for the electrons' movement and that the two regions around He nuclei form a double-well-like potential. The LRF of $\mathrm{He}_{2}{ }^{2+}$ indicated by the equivalue surfaces, $\delta \rho(\mathbf{r}) / \delta \mathrm{v}(\mathrm{X})= \pm 0.1$, is shown in Figure $7 \mathrm{~b}$, which is, in fact, similar to that of Figures 5a and $6 \mathrm{a}$. A reason why the amplitudes of LRF of $\mathrm{He}_{2}{ }^{2+}$ become larger than those of $\mathrm{H}_{2}$ is that the HOMO and LUMO are degenerate as in the case of double-well potential with Nocc $=1$. In fact, the HOMO-LUMO gap of $\mathrm{He}_{2}{ }^{2+}$ with this geometry, 0.0367 Hartree, is one order of magnitude smaller than that of $\mathrm{H}_{2}$, resulting in the larger amplitude of LRF. Since the phases of HOMO and LUMO of $\mathrm{He}_{2}{ }^{2+}$ are similar to those of $\mathrm{H}_{2}$, the LRF of $\mathrm{He}_{2}{ }^{2+}$ is qualitatively similar to that of $\mathrm{H}_{2}$ as obviously observed from Figure 7a,b.

Next, we calculated LRF of $\mathrm{He}_{2}$ with the interatomic distance, $\mathrm{R}=3.0 \AA$. As shown in Figure 7c, the equivalue surfaces show no amplitude even if we set $\delta \rho(r) / \delta v(X)= \pm 0.001$. In fact, the maximum value of $\delta \rho(\mathbf{r}) / \delta \mathrm{v}(\mu)$ is \pm 0.00001 , which is a result of large HOMO-LUMO gap (1.745 Hartree). In the Section 3.1.1, this LRF is similar to those shown in Figure 5b,d, and Figure 6b,d,f: NEM of small numbers of electrons holds for closed shell systems without any covalent bond.

Figure 7. Equivalue surfaces of linear response functions for (a) $\mathrm{H}_{2}$, (b) $\mathrm{He}_{2}{ }^{2+}$, and (c) $\mathrm{He}_{2}$. The threshold of isosurfaces are (a) $\delta \rho(\mathbf{r}) / \delta \mathrm{v}(\mathrm{X})= \pm 0.01$; (b) $\delta \rho(\mathbf{r}) / \delta \mathrm{v}(\mathrm{X})= \pm 0.1$, and (c) $\delta \rho(r) / \delta v(X)= \pm 0.001$, respectively.

(a)

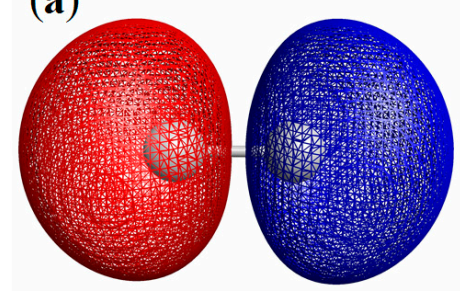

(b)

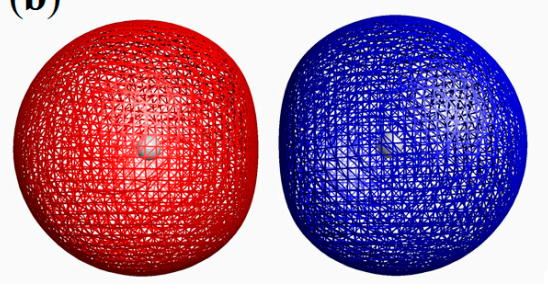

(c)

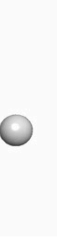

3.2.2. Dissociation of the Hydrogen Molecule: A Possible Mott-insulator Counterpart to NEM

From the comparison between LRFs of $\mathrm{He}_{2}$ and $\mathrm{He}_{2}{ }^{2+}$, the energy gap between highest occupied and lowest unoccupied orbitals seems to be closely related to NEM of the systems. In fact, the 
magnitude of the linear response varies along the dissociation profile of covalent bonds, as was previously reported $[25,26]$. We will describe it again here for completeness of our discussion.

For this purpose, we present LRF of $\mathrm{H}_{2}$ molecule with various interatomic distances that are obtained from spin-restricted (R) and spin-unrestricted B3LYP (UB3LYP) solutions. Figure 8 shows the magnitude of LRF, which is defined as $\int \mathrm{d} \mathbf{r}|\delta \rho(\mathbf{r}) / \delta \mathrm{v}(\mathrm{X})|$ with $\mathrm{X}$ being the right-atom as in the previous section, for the dissociation profile. We can see from this figure that the magnitude of LRF monotonically increases as the interatomic distance $(\mathrm{R})$ increases for RB3LYP solutions. This is because the HOMO-LUMO gap in the denominator of the right-hand of Equation (15) decreases as the interatomic distance increases. For $\mathrm{R}<1.5 \AA$, UB3LYP solutions are the same as the RB3LYP solutions. However, for $\mathrm{R} \geq 1.5 \AA$, the magnitude of LRF decreases for UB3LYP solutions. This can be explained as follows. LRF of UB3LYP solutions is a sum of $\alpha$ and $\beta$ parts:

$$
\frac{\delta \rho(\mathbf{r})}{\delta \mathrm{v}\left(\mathbf{r}^{\prime}\right)} \cong \sum_{\sigma}^{\alpha, \beta} \frac{\psi_{\mathrm{HOMO}}^{\sigma}(\mathbf{r}) \psi_{\mathrm{HOMO}}^{\sigma}\left(\mathbf{r}^{\prime}\right) \psi_{\mathrm{LUMO}}^{\sigma}(\mathbf{r}) \psi_{\mathrm{LUMO}}^{\sigma}\left(\mathbf{r}^{\prime}\right)}{\varepsilon_{\mathrm{HOMO}}^{\sigma}-\varepsilon_{\mathrm{LUMO}}^{\sigma}}
$$

In the manner similar to the RB3LYP case, the HOMO-LUMO gap decreases as R increases. However, beyond $\mathrm{R}=1.5 \AA$, the chemical bond breaks and $\alpha$ and $\beta$ orbitals start to localize to the opposite sites in the hydrogen molecule. At the dissociation limit, they reduce to the atomic orbitals of opposite sides of the hydrogen molecule as:

$$
\begin{aligned}
& \psi_{\mathrm{HOMO}}^{\alpha}(\mathbf{r}) \rightarrow \chi_{1}^{\alpha}(\mathbf{r}), \psi_{\mathrm{LUMO}}^{\alpha}(\mathbf{r}) \rightarrow \chi_{2}^{\alpha}(\mathbf{r}) \\
& \psi_{\mathrm{HOMO}}^{\beta}(\mathbf{r}) \rightarrow \chi_{2}^{\beta}(\mathbf{r}), \psi_{\mathrm{LUMO}}^{\beta}(\mathbf{r}) \rightarrow \chi_{2}^{\beta}(\mathbf{r})
\end{aligned}
$$

Figure 8. Interatomic distance of $\mathrm{H}_{2}$ versus magnitude of $\operatorname{LRF}\left(\int \mathrm{d} \mathbf{r}|\delta \rho(\mathbf{r}) / \delta \mathrm{v}(\mathrm{X})|\right)$.

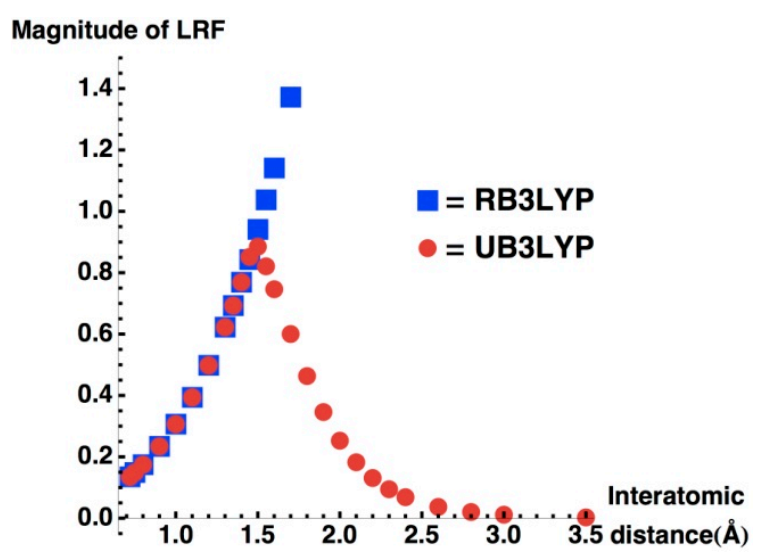

Thus, the numerators of the right side of Equation (16) converge to zero more rapidly than the denominator diverges, and consequently the magnitude of LRF reduces to zero as R increases. In order to make the situation clear, we plotted equivalue surfaces of LRF of UB3LYP solutions for $R=0.8$, $1.5,2.2,2.5$, and $3.5 \AA$ in Figure 9. A noteworthy point is that the LRF for $\mathrm{R}=3.5 \AA$ vanishes for this resolution, implying that the nearsightedness holds for the dissociated limit. Obviously, this is because occupied orbitals localize as described in Equation (17), which is parallel to the fundamental feature of the insulating state that was described by Kohn [5]. As for molecular systems, this could be the origin of NEM for molecular magnetic systems such as diradical and polyradical systems [29]. However we should note that usual closed-shell systems such as organic and inorganic stable compounds do not 
usually exhibit such magnetism. In the next subsection, we shall disscuss an origin of NEM for usual molecular systems.

Figure 9. Equivalue surfaces of LRF of UB3LYP solutions for various interatomic distances (Rs). The threshold is similar to that of Figure $7 \mathrm{a}(\delta \rho(\mathbf{r}) / \delta \mathrm{v}(\mathrm{X})= \pm 0.01)$. (a) $\mathrm{R}=0.8 \AA$; (b) $\mathrm{R}=1.5 \AA$; (c) $\mathrm{R}=2.2 \AA$; (d) $\mathrm{R}=2.5 \AA$, and (e) $\mathrm{R}=3.5 \AA$.
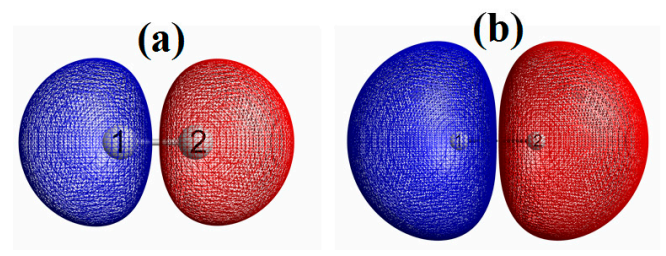

(c)

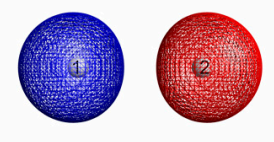

(d)

(e)

\subsubsection{Trialanine Peptide System: $\mathrm{sp}_{3}$ Junctions as a Possible Origin of NEM of Molecular Systems}

Finally, we calculated LRF of trialanine peptide system with $\alpha$-helix structure in order to show whether and how the nearsightedness holds for usual molecular systems that are treated with QM/MM calculations. The quasi-equilibrium geometry at $300 \mathrm{~K}$ is obtained with using the Amber molecular dynamics simulations program [30]. The umbrella sampling [31] with WHAM scheme [32] is employed. Computational details are described in Section S3 of the Supplementary Material. The geometry is illustrated in Figure 10.

Figure 10. The selected geometry of the a-helix structure of trialanine peptide system.

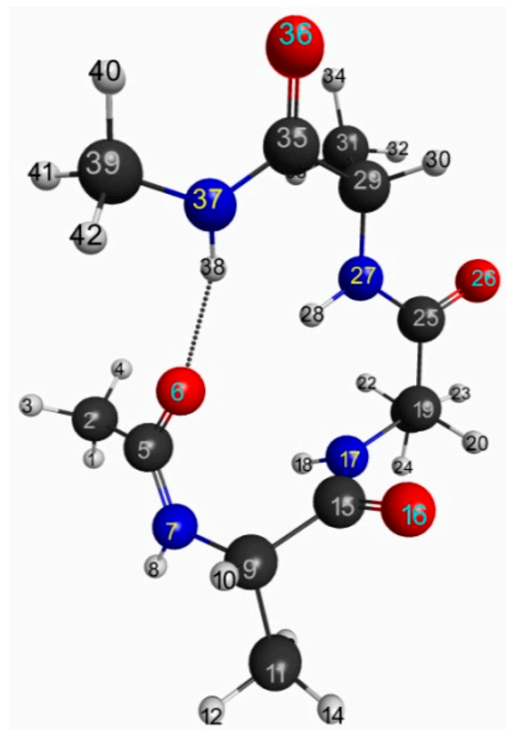

The isosurfaces of the LRFs to the perturbations that are applied to the atomic sites are shown in Figure S2 of the Supplementary Material. We selected a few isosurface plots in Figure 11. Here the isosurfaces for $\delta \rho(\mathbf{r}) / \delta \mathrm{v}_{\mathrm{X}}=0.01$ and $=-0.01$ are described as blue and red surfaces, respectively. This threshold is similar to that of $\mathrm{H}_{2}$ shown in Figure 7a. The number described below each isosurface corresponds to the atom number shown in Figure 10. From these plots (and more comprehensive plots shown in Figure S2), we can see that the density deviations do not propagate over the other side of the $\mathrm{C}_{\alpha}$ atom: for instance, if we perturb the oxygen atom of carbonyl group (the atom number is 6 ), the 
effects localize within the amide plane. We can see similar behaviors in 5, 6, 7, 14, 15, 16, 28, 37, 38, 42 in Figure 11.

In other words, the sp3 junctions block the propagations of the errors of the QM/MM modeling. In addition, it was also observed that the LRFs do not propagate via the hydrogen bond of the $\alpha$ helix structure between $\mathrm{O}(6)$ and $\mathrm{H}(38)$. Further, the perturbations on hydrogen atoms of the methyl group $(14,42)$ do not affect the main chain. In other words, NEM holds for this system. However, we should note that the mechanism to exhibit NEM is different from those of simple model systems, which are shown in Figures 1i, 5i and 6i. In this system, the sp3 junction seems to be the main cause to prevent the propagation of LRFs, which is caused by an orbital dependent mechanism rather than by a destructive interference among density amplitudes [4].

In order to more clearly exemplify this " $\mathrm{sp}_{3}$ junction mechanism" for NEM of molecular systems, we considered more simple systems: butane and butadiene molecules. We showed the LRFs of butane and butadiene in Figure 12a,b, respectively. Here the perturbation is applied to the right terminal C atom for both molecules (we listed all other plots for the LRFs, in which the perturbation are applied for other atoms, in Figures S3 and S4, respectively, for butane and butadiene). Obviously, the density deviations propagate over the whole carbon framework via the $\pi$-conjugated channel for the butadiene case, while the density deviations are blocked with the $\mathrm{sp}_{3}$ junction at the carbon atom next to the terminal carbon atom. This is why the boundaries for QM/MM calculations should be set on single bonds, but not on $\pi$-bonds $[25,26]$.

Figure 11. Isosurfaces of linear response functions for the perturbations on atomic sites. $\delta \rho(\mathbf{r}) / \delta \mathrm{v}_{\mathrm{X}}=0.01$ and $=-0.01$ are indicated as blue and red surfaces, respectively. For carbons and hydrogens, we indicate the atomic types as follows. $\mathrm{H}\left(\mathrm{C}_{\mathrm{T}}\right)=\mathrm{H}$ of the terminal methyl group. $\mathrm{H}\left(\mathrm{C}_{\alpha}\right)=\mathrm{H}$ in the amide plane. $\mathrm{H}\left(\mathrm{C}_{\beta}\right)=\mathrm{H}$ of the methyl residue. $\mathrm{C}_{\beta}$, and $\mathrm{C}(0)$ are $\beta$ carbon and carbon of the carbonyl group, respectively.

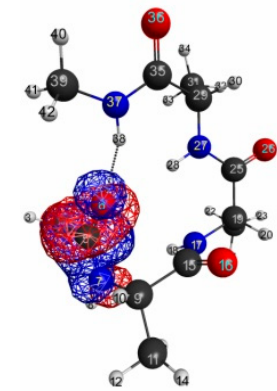

$5 \mathrm{C}(\mathrm{O})$

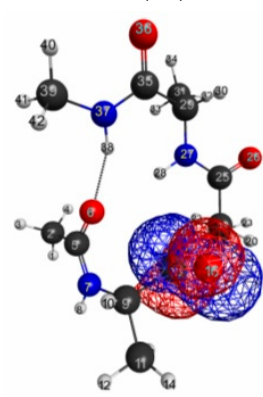

$16 \mathrm{O}$

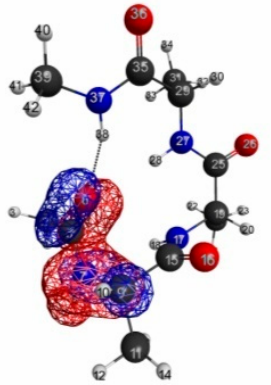

$6 \mathrm{O}$

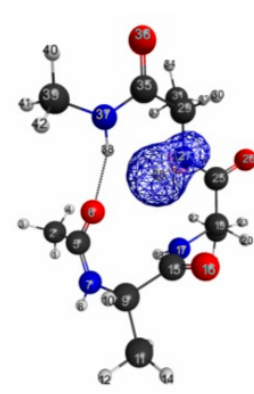

$28 \mathrm{H}(\mathrm{N})$

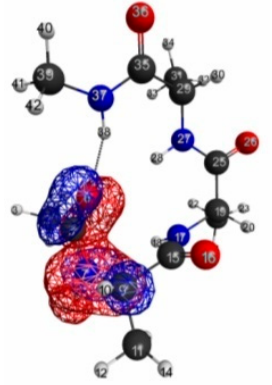

$7 \mathrm{~N}$

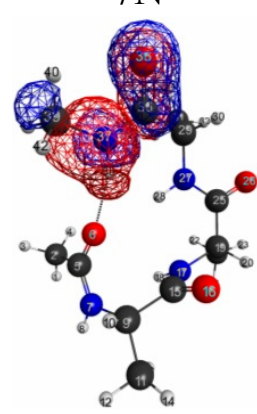

$37 \mathrm{~N}$

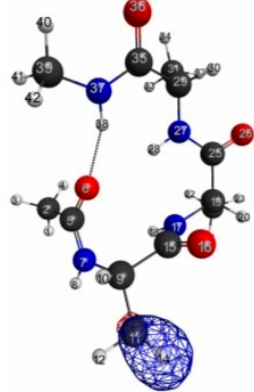

$14 \mathrm{H}\left(\mathrm{C}_{\beta}\right)$

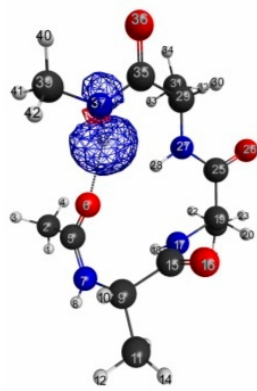

$38 \mathrm{H}(\mathrm{N})$

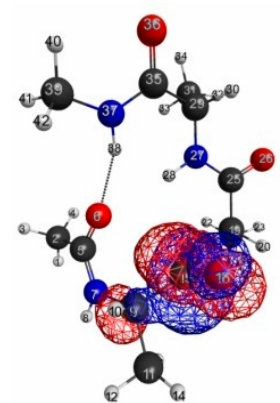

$15 \mathrm{C}(\mathrm{O})$

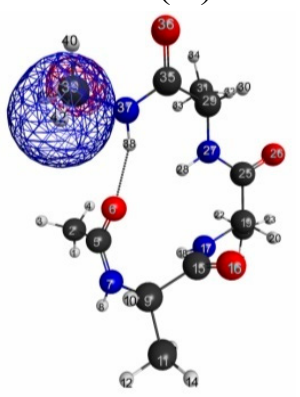

$42 \mathrm{H}\left(\mathrm{C}_{\mathrm{T}}\right)$ 
Figure 12. Isosurfaces of linear response functions for the perturbations on the right terminal $\mathrm{C}$ atoms. $\delta \rho(\mathbf{r}) / \delta \mathrm{v}_{\mathrm{X}}=0.035$ and $=-0.035$ are presented as blue and red surfaces, respectively; (a) butane and (b) butadiene.

(a)

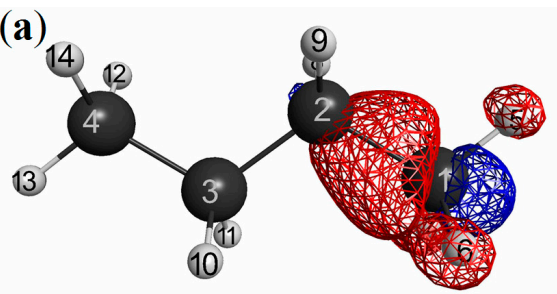

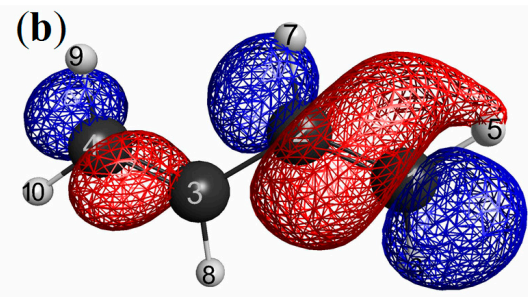

\section{Conclusions}

We examined nearsightedness of electronic matter (NEM) of finite model and molecular systems on the basis of linear response function (LRF). From the computational results of both the single-and-double square-well model systems, we found that, as $\mathrm{N}$ increases, LRF, $\delta \rho(\mathrm{r}) / \delta \mathrm{v}\left(\mathrm{r}^{\prime}\right)$, decays rapidly for the distance, $\left|r-r^{\prime}\right|$, being consistent with the principle suggested by Kohn and Prodan: the cause of NEM is destructive interference among electron density amplitudes [4]. For a few electrons systems of double-well model systems, we observed that there are two cases: the cases of the half-filled and of full-filled in low-lying degenerate levels. The response for the former is delocalized, while that of the later is localized even if the systems contain only a few electrons. These behaviors are discussed in relation to the molecular systems' counterparts, $\mathrm{H}_{2}, \mathrm{He}_{2}{ }^{2+}$, and $\mathrm{He}_{2}$ systems. In addition, we also present that NEM holds for dissociated $\mathrm{H}_{2}$ because of the localized feature of $\alpha$ and $\beta$ electrons, which is essentially similar to that of insulating states [5]. Finally, we examined LRF of alanine tripeptide system as well as butane and butadiene systems, and found that NEM of these organic systems is mainly caused by $\mathrm{sp}_{3}$ junctions at Cas that prevent propagation of amplitudes of LRF. In other words, the cause of NEM of these organic systems is critically different from that of NEM for finite and infinite homogeneous systems.

\section{Supplementary Materials}

Supplementary materials can be accessed at: http://www.mdpi.com/1420-3049/19/9/13358/s1.

\section{Acknowledgments}

This work is partly supported by a grant from Japan Science and Technology Agency (JST) with a Core Research for Evolutional Science and Technology (CREST).

\section{Author Contributions}

Shusuke Yamanaka, the corresponding author, was in charge of the theoretical part of this work, provided ideas as well as computational codes, performed a part of calculations, and wrote this paper. Yuki Mitsuta implemented the linear response analyses of model systems using his Mathematica code. Mitsutaka Okumura and Kizashi Yamaguchi provided guidance and suggestions during this work. 
Haruki Nakamura is a coordinator of this series of studies based on linear response function analysis, in particular that for the $\mathrm{QM} / \mathrm{MM}$ boundary problem, which is the original motivation of this study.

\section{Conflicts of Interest}

The authors declare no conflict of interest.

\section{References and Notes}

1. Warshel, A.; Karplus, M. Calculation of ground and excited state potential surfaces of conjugated molecules. I. Formulation and parametrization. J. Am. Chem. Soc. 1972, 94, 5612-5625.

2. Warshel, A.; Levitt, M. Theoretical studies of enzymic reactions: Dielectric, electrostatic and steric stabilization of the carbonium ion in the reaction of lysozyme. J. Mol. Biol. 1976, 103, 227-249.

3. Kohn, W. Density functional and density matrix method scaling linearly with the number of atoms. Phys. Rev. Lett. 1996, 76, 3168-3171.

4. Prodan, E.; Kohn, W. Nearsightedness of electronic matter. Proc. Natl. Acad. Sci. USA 2005, 112, 11635-11638.

5. Kohn, W. Theory of insulating states. Phys. Rev. 1964, 133, A171-A181.

6. Hohenberg, P.; Kohn, W. Inhomogeneous electron gas. Phys. Rev. 1964, 136, B864-B871.

7. Kohn, W.; Sham, L.-J. Self-consistent equations including exchange and correlation effects. Phys. Rev. 1965, 140, A1133-A1138.

8. Feynmann, R.P. Forces in molecules. Phys. Rev. 1939, 56, 340-343.

9. Sukumar, M. A Matter of Density: Exploring the Electron Density Concept in the Chemical, Biological, and Materials Sciences; John-Wiley \& Sons: Hoboken, NJ, USA, 2013.

10. Friedel, J. The distribution of electrons round impurities in monovalent metals. Philos. Mag. 1952, 43, 153-189.

11. Langer, J.S.; Vosko, S.H. The shielding of a fixed charge in a high-density electron gas. Philos. Mag. 1952, 43, 153-189.

12. Kohn, W.; Vosko S.H. Theory of nuclear resonance intensity in dilute alloys. Phys. Rev. 1960, 119, 912-918.

13. Ono, M.; Nishigata, Y.; Nishio, T.; Eguchi, T.; Hasegawa, Y. Electrostatic potential screened by a two-dimensional electron system: A real-space observation by scanning tunneling spectroscopy. Phys. Rev. Lett. 2006, 96, 016801:1-016801:4.

14. Fetter, A.L.; Walecka, J.D. Quantum Theory of Many-Particle Systems, 3rd ed.; Dover Pubications: Mineola, NY, USA, 2003; pp. 171-183.

15. Ayer, P.W.; Parr, R.G. Variational principles for describing chemical reactions. Reactivity indices based on the external potential. J. Am. Chem. Soc. 2001, 123, 2007-2015.

16. Geerlings, P.; Proft, F.D.; Langenaeker, W. Conceptual density functional theory. Chem. Rev. 2003, 103, 1793-1873.

17. Berkowitz, M.; Parr, R.G. Molecular hardness and softness, local hardness and softness, hardness and softness kernels, and relations among these quantities. J. Chem. Phys. 1988, 88, 2554-2557.

18. Senet, P.J. Nonlinear electronic responses, Fukui functions and hardnesses as functionals of the groundstate electronic density. J. Chem. Phys. 1996, 105, 6471-6489. 
19. Sablon, N.; Proft, F.D.; Geerlings, P. The linear response kernel: Inductive and resonance effects quantified. J. Phys. Chem. Lett. 2010, 1, 1228-1234.

20. Gonzalez-Suarez, M.; Aizman, A.; Contreras, R. Phenomenological chemical reactivity theory for mobile electrons. Theor. Chem. Acc. 2010, 126, 45-54.

21. Mitito E.; Putz, M.V. New Link between conceptual density functional theory and electron delocalization. J. Phys. Chem. A 2011, 115, 12459-12462.

22. Putz, M.V.; Chattaraj, P.K. Electrophilicity kernel and its hierarchy through softness in conceptual density functional theory. Int. J. Quantum Chem. 2013, 113, 2163-2171.

23. Schiff, L.I. Quantum Mechanics, 3rd ed.; McGraw-Hill: New York, NY, USA, 1968.

24. Mathematica Ver. 9, Wolfram Research: Champaign, IL, USA. Available online: http://www.wolfram.com/mathematica (accessed on 25 August 2014).

25. Yamanaka, S.; Yonezawa, Y.; Nakata, K.; Nishihara, S.; Okumura, M.; Takada, T.; Yamaguchi, K.; Nakamura, H. Locality and nonlocality of electronic structures of molecular systems: Toward QM/MM and QM/QM approaches. AIP Conf. Proc. 2012, 1504, 916-919.

26. Ueda, K.; Yamanaka, S.; Nakata, K.; Ehara, M.; Okumura, M.; Yamaguchi, K.; Nakamura, H. Linear response function approach for the boundary problem of QM/MM methods. Int. J. Quantum Chem. 2013, 113, 336-341.

27. Schmidt, M.W.; Baldridge, K.K.; Boatz, J.A.; Elbert, S.T.; Gordon, M.S.; Jensen, J.H.; Koseki, S.; Matsunaga, N.; Nguyen, K.A.; Su, S.; et al. General atomic and molecular electronic structure system. J. Comput. Chem. 1993, 14, 1347-1363.

28. Becke, A.D. Density-functional thermochemistry. III. The role of exact exchange. J. Chem. Phys. 1993, $98,5648-5652$.

29. Yamaguchi, K.; Jensen, F.; Dorigo, A.; Houk, K.N. A spin correction procedure for unrestricted Hartree-Fock and Moller-Plesset wavefunctions for singlet diradicals and polyradicals. Chem. Phys. Lett. 1988, 149, 537-542.

30. Salomon-Ferrer, R.D.; Case, A.; Walker, R.C. An overview of the Amber biomolecular simulation package. WIREs Comput. Mol. Sci. 2013, 3, 198-210.

31. Torrie, G.M.; Valleau, J.P. Nonphysical sampling distributions in Monte Carlo free-energy estimation: Umbrella sampling. J. Comput. Phys. 1977, 23, 187-199.

32. Kumar, S.; Bouzida, D.; Swendsen, R.H.; Kollman, P.A.; Rosenberg, J.M. THE weighted histogram analysis method for free-energy calculations on biomolecules. I. The method. J. Comput. Chem. 1992, 13, 1011-1021.

Sample Availability: Not available.

(C) 2014 by the authors; licensee MDPI, Basel, Switzerland. This article is an open access article distributed under the terms and conditions of the Creative Commons Attribution license (http://creativecommons.org/licenses/by/3.0/). 